\title{
A VARIATIONAL APPROACH FOR A BI-NON-LOCAL ELLIPTIC PROBLEM INVOLVING THE $p(x)$-LAPLACIAN AND NON-LINEARITY WITH NON-STANDARD GROWTH
}

\author{
FRANCISCO JULIO S. A. CORRÊA \\ Centro de Ciências e Tecnologia, \\ Unidade Acadêmica de Matemática e Estatística, Universidade Federal de Campina Grande, \\ CEP:58.109-970, Campina Grande, Parā̄ba, Brazil \\ e-mail: fjsacorrea@gmail.com \\ and AUGUSTO CÉSAR DOS REIS COSTA \\ Instituto de Ciências Exatas e Naturais, \\ Faculdade de Matemática, Universidade Federal do Pará, CEP:66075-110, Belém, Pará, Brazil \\ e-mail:aug@ufpa.br
}

(Received 20 September 2012; revised 29 January 2013; accepted 10 May 2013; first published online 20 August 2013)

\begin{abstract}
In this paper we are concerned with a class of $p(x)$-Kirchhoff equation where the non-linearity has non-standard growth and contains a bi-non-local term. We prove, by using variational methods (Mountain Pass Theorem and Ekeland Variational Principle), several results on the existence of positive solutions.
\end{abstract}

2000 Mathematics Subject Classification. 35J60; 35J70; 58E05.

1. Introduction. In this paper we investigate questions of existence of positive solutions for the bi-non-local elliptic problem

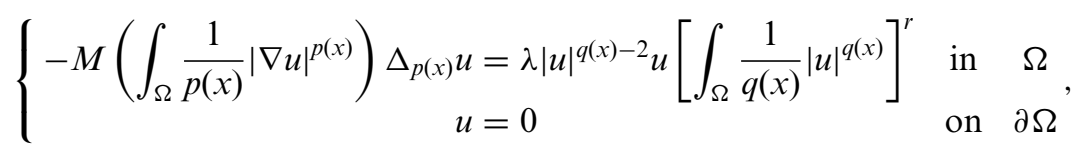

where $\Omega \subset \mathbb{R}^{N}$ is a bounded smooth domain, $\lambda, r>0$, are real parameters and $M$ : $\mathbb{R}^{+} \rightarrow \mathbb{R}^{+}$is a continuous function, $p, q \in C(\bar{\Omega})$ are functions whose properties will be given later and $\Delta_{p(x)} u=\operatorname{div}\left(|\nabla u|^{p(x)-2} \nabla u\right)$ is the $p(x)$-Laplacian.

Problem (1.1) is a version of the non-local problem

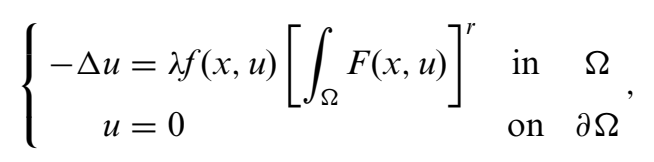

with $F(t)=\int_{0}^{t} f(s) d s$, where $f: \mathbb{R} \rightarrow \mathbb{R}$ is a given regular function.

Problems in the form (1.1) are associated with the energy functional

$$
J_{\lambda}(u)=\widehat{M}\left(\int_{\Omega} \frac{1}{p(x)}|\nabla u|^{p(x)}\right)-\lambda \frac{1}{r+1}\left[\int_{\Omega} \frac{1}{q(x)}|u|^{q(x)}\right]^{r+1}
$$


for all $u \in W_{0}^{1, p(x)}(\Omega)$, where $W_{0}^{1, p(x)}(\Omega)$ is the generalised Lebesgue-Sobolev space whose precise definition and properties will be established in Section 1 and $\widehat{M}(t)=$ $\int_{0}^{t} M(s) d s$

Depending on the behaviour of the functions $p, q$, the above functional is differentiable and its Fréchet-derivative is given by

$$
J_{\lambda}^{\prime}(u) v=M\left(\int_{\Omega} \frac{1}{p(x)}|\nabla u|^{p(x)}\right) \int_{\Omega}|\nabla u|^{p(x)-2} \nabla u \nabla v-\lambda\left[\int_{\Omega} \frac{1}{q(x)}|u|^{q(x)}\right]^{r} \int_{\Omega}|u|^{q(x)-2} u v
$$

for all $u, v \in W_{0}^{1, p(x)}(\Omega)$. So accordingly the definition that we give in the next section, $u \in W_{0}^{1, p(x)}(\Omega)$, is a weak solution of problem (1.1) if and only if $u$ is a critical point of $J_{\lambda}$.

Before giving preliminaries on the space $W_{0}^{1, p(x)}(\Omega)$ and on the operator $\Delta_{p(x)}$, we give some motivation for the study of problems (1.1) and (1.2).

Indeed, problems like (1.2) have been previously studied by several authors. Let us cite some of them.

Gomes and Sanchez [16] studied problem (1.2) by using a variational approach by considering $f$ with a sort of exponential growth and $\Omega$ as a ball of $\mathbb{R}^{N}$. In this work, the authors improve results contained in Bebernes and Lacey [3].

Bebernes and Talaga in [4] considered a particular case of (1.2), namely

$$
\left\{\begin{array}{rlrl}
-\Delta u & =\delta \frac{e^{u}}{\int_{\Omega} e^{u}} & \text { in } \quad & \Omega \\
u & =0 & \text { on } & \partial \Omega
\end{array},\right.
$$

with $f(t)=F(t)=e^{t}$, which is the stationary counterpart of the parabolic problem

$$
\left\{\begin{array}{rlrrrr}
u_{t}-\Delta u & =\delta \frac{e^{u}}{\int_{\Omega} e^{u}} & & \text { in } & & \Omega \times[0, T) \\
u(x, 0) & =u_{0}(x) & & \text { in } & & \Omega \\
u(x, t) & =0 & & \text { on } & & \partial \Omega \times[0, T)
\end{array},\right.
$$

which arises in the analytical investigation of phenomena associated with the occurrence of shear bands in metals being deformed under high strain rates. See also Burns $([\mathbf{5}, \mathbf{6}])$ and Olmstead et al. [20]. It also arises in the investigation of a fully turbulent behaviour of a real flow and in the theory of gravitational equilibrium of polytropic stars. See, for example, Caglioti et al. [7], and Krzywick and Nadzieja [17].

Other physical motivations for the problem (1.2) may be found in $[\mathbf{8}, \mathbf{1 0}, \mathbf{1 5}]$ and the references therein. These works are related with problem (1.2), in which $p$ is constant and so its study is done in the usual Sobolev space. However, we are concerned with problem (1.1) in which the $p(x)$-Laplace operator appears and the growth of nonlinearities are not standard, i.e. the exponent $q(x)$ depends on $x \in \bar{\Omega}$.

This type of problem has interesting motivation from both physical and mathematical point of view. Indeed, it appears in the so-called model of motion of electrorheological fluids, characterized by their ability to change in a drastic way the mechanical properties when influenced by an exterior electromagnetic field. In order to emphasize the importance of this subject, we cite Mihailescu and Radulescu [18]: The first major discovery on electrorheological fluids is due to Willis Winslow in 1949. These fluids have the interesting property that their viscosity depends on the electric 
field in the fluid. He noticed that in such fluids (for instance lithium polymetachrylate) viscosity in a electrical field is inversely proportional to the strength of the field. The field induces string-like formations in the fluid, which are parallel to the field. They can raise the viscosity by as much as five orders of magnitude. This phenomenon is known as the Winslow effect. Electrorheological fluids has been used in robotic and space technology. The experimental research has been done mainly in the USA, for instance in NASA laboratories.

For more information on this subject, the reader may consult, for example, [18] and the references therein.

Another application of such a kind of equation is related to image processing. See [23] and the references therein.

Besides these practical applications, there is the mathematical aspect that involves nontrivial questions of the generalized Lebesgue-Sobolev spaces and the $p(x)$ Laplacian operator. In Section 2 we give a review of some concepts and results on this subject necessary for the development of this work.

With respect to the Kirchhoff equation, most of the works treat problems like

$$
\left\{\begin{array}{rl}
-M\left(\|u\|_{1, p}^{p}\right) \Delta_{p} u & =f(x, u) \text { in } \Omega \\
u & =0 \text { on } \partial \Omega
\end{array},\right.
$$

where $M$ is the original Kirchhoff term of the form $M(t)=a+b t, t \geq 0, a, b>0$ and $1 \leq p<+\infty$ are real constants. In Alves et al. [2] and Corrêa and Figueiredo [9] other $M$ terms were considered.

We also cite [21] and [22], in which the authors study

$$
\left\{\begin{aligned}
-\|u\|^{2} \Delta u & =\mu u^{3} \text { in } \Omega, \\
u & =0 \text { on } \partial \Omega
\end{aligned}\right.
$$

as an auxiliary problem where $\|u\|=\left(\int_{\Omega}|\nabla u|^{2}\right)^{1 / 2}$ is the usual norm of $H_{0}^{1}(\Omega)$.

Note that in Equation (1.2) only the non-local term $\left[\int_{\Omega} F(x, u)\right]^{r}$ appears, while in Equation (1.6) the only non-local term is $M\left(\|u\|_{1, p}^{p}\right)$.

We should point out that the literature related to problems like (1.1), even for the case of $p \equiv 2$, is limited. We cite, for instance, Agarwal et al. [1], in which the authors focus their attention on the problem

$$
\left\{\begin{aligned}
-\|u\|_{1, q}^{p-q} \Delta_{q} u & =\lambda\|u\|_{r}^{p-r}|u|^{r-2} u \text { in } \Omega \\
u & =0 \text { on } \partial \Omega
\end{aligned}\right.
$$

where $\|u\|_{1, q}=\left(\int_{\Omega}|u|^{q}\right)^{1 / q}$ is the usual norm in $W_{0}^{1, q}(\Omega)$.

The novelty in this paper, at least to our knowledge, is to relate the bi-nonlocal problem (1.1), with the variable exponent theory, which possesses a variational structure. Using solely variational methods (Mountain Pass Theorem and Ekeland Variational Principle), we establish several results by considering various classes of the Kirchhoff term $M$ and ranges determined by $p, q, M, r$ and $\lambda$. We only consider the subcritical case. The critical case will be attacked in a forthcoming paper.

This work is organized as follows: In Section 2 we establish some preliminary results related to the generalized Lebesgue-Sobolev spaces and the $p(x)$-Laplacian. In Section 3 we prove the existence results. 
2. Preliminary results. First of all we set

$$
C_{+}(\bar{\Omega})=\{h ; h \in C(\bar{\Omega}), h(x)>1 \text { for all } x \in \bar{\Omega}\}
$$

and for each $h \in C_{+}(\bar{\Omega})$ we define

$$
h^{+}=\max _{\bar{\Omega}} h(x) \text { and } h^{-}=\min _{\bar{\Omega}} h(x) .
$$

We denote by $\mathcal{M}(\Omega)$ the set of real measurable functions defined on $\Omega$.

For each $p \in C_{+}(\bar{\Omega})$, we define the generalized Lebesgue space by

$$
L^{p(x)}(\Omega)=\left\{u \in \mathcal{M}(\Omega) ; \int_{\Omega}|u(x)|^{p(x)} d x<\infty\right\} .
$$

We consider $L^{p(x)}(\Omega)$ equipped with the Luxemburg norm,

$$
|u|_{p(x)}=\inf \left\{\mu>0 ; \int_{\Omega}\left|\frac{u(x)}{\mu}\right|^{p(x)} d x \leq 1\right\} .
$$

The generalized Lebesgue-Sobolev space $W^{1, p(x)}(\Omega)$ is defined by

$$
W^{1, p(x)}(\Omega)=\left\{u \in L^{p(x)}(\Omega) ;|\nabla u| \in L^{p(x)}(\Omega)\right\}
$$

with the norm

$$
\|u\|_{1, p(x)}=|u|_{p(x)}+|\nabla u|_{p(x)} .
$$

We define $W_{0}^{1, p(x)}(\Omega)$ as being the closure of $C_{c}^{\infty}(\Omega)$ in $W^{1, p(x)}(\Omega)$ with respect to the norm $\|u\|_{1, p(x)}$. According to Fan and Zhao [13], the spaces $L^{p(x)}(\Omega), W^{1, p(x)}(\Omega)$ and $W_{0}^{1, p(x)}(\Omega)$ are separable and reflexive Banach spaces. Furthermore, if the Lebesgue measure of $\Omega$ is finite, $p_{1}, p_{2} \in C(\bar{\Omega})$ and $p_{1}(x) \leq p_{2}(x)$, for all $x \in \Omega$, then we have the continuous embedding $L^{p_{2}(x)}(\Omega) \hookrightarrow L^{p_{1}(x)}(\Omega)$.

The proof of the following propositions may be found in [11-13].

Proposition 2.1. Suppose that $\Omega$ is a bounded smooth domain in $\mathbb{R}^{N}$ and $p \in C(\bar{\Omega})$ with $p(x)<N$ for all $x \in \bar{\Omega}$. If $p_{1} \in C(\bar{\Omega})$ and $1 \leq p_{1}(x) \leq p^{*}(x)\left(1 \leq p_{1}(x)<p^{*}(x)\right)$ for $x \in \bar{\Omega}$, then there is a continuous (compact) embedding $W^{1, p(x)}(\Omega) \hookrightarrow L^{p_{1}(x)}(\Omega)$, where $p^{*}(x)=\frac{N p(x)}{N-p(x)}$.

Proposition 2.2. Set $\rho(u)=\int_{\Omega}|u(x)|^{p(x)} d x$. For all $u \in L^{p(x)}(\Omega)$, we have:

(1) For $u \neq 0,|u|_{p(x)}=\lambda \Leftrightarrow \rho\left(\frac{u}{\lambda}\right)=1$.

(2) $|u|_{p(x)}<1(=1 ;>1) \Leftrightarrow \rho(u)<1(=1 ;>1)$.

(3) If $|u|_{p(x)}>1$, then $|u|_{p(x)}^{p^{-}} \leq \rho(u) \leq|u|_{p(x)}^{p^{+}}$.

(4) If $|u|_{p(x)}<1$, then $|u|_{p(x)}^{p^{+}} \leq \rho(u) \leq|u|_{p(x)}^{p^{-}}$.

(5) $\lim _{k \rightarrow+\infty}\left|u_{k}\right|_{p(x)}=0 \Leftrightarrow \lim _{k \rightarrow+\infty} \rho\left(u_{k}\right)=0$.

(6) $\lim _{k \rightarrow+\infty}\left|u_{k}\right|_{p(x)}=+\infty \Leftrightarrow \lim _{k \rightarrow+\infty} \rho\left(u_{k}\right)=+\infty$. 
Proposition 2.3 (Poincaré Inequality). If $u \in W_{0}^{1, p(x)}(\Omega)$, then

$$
|u|_{p(x)} \leq C|\nabla u|_{p(x)},
$$

where $C$ is a constant that does not depend on $u$.

Note that, in view of Poincare inequality, the norms $\|\cdot\|_{1, p(x)}$ and $\|u\|=|\nabla u|_{p(x)}$ are equivalent in $W_{0}^{1, p(x)}(\Omega)$. From now on we work on $W_{0}^{1, p(x)}(\Omega)$ with the norm $\|u\|=|\nabla u|_{p(x)}$.

We denote by $L^{p^{\prime}(x)}(\Omega)$ the conjugate space of $L^{p(x)}(\Omega)$, where

$$
\frac{1}{p(x)}+\frac{1}{p^{\prime}(x)}=1, \text { for all } x \in \bar{\Omega} .
$$

Proposition 2.4 (Hölder Inequality). If $u \in L^{p(x)}(\Omega)$ and $v \in L^{p^{\prime}(x)}(\Omega)$, then

$$
\left|\int_{\Omega} u v d x\right| \leq\left(\frac{1}{p^{-}}+\frac{1}{p^{\prime-}}\right)|u|_{p(x)}|v|_{p^{\prime}(x)} .
$$

In what follows, we consider the problem

$$
\left\{\begin{aligned}
-M\left(\int_{\Omega} \frac{1}{p(x)}|\nabla u|^{p(x)}\right) \Delta_{p(x)} u & =\lambda\left(u^{+}\right)^{q(x)-1}\left[\int_{\Omega} \frac{1}{q(x)}\left(u^{+}\right)^{q(x)}\right]^{r} \text { in } \Omega, \\
u & =0
\end{aligned}\right.
$$

because we are interesting in finding positive solutions. Note that possible solutions of (2.7) are positive solutions of (1.1). See Fan et al. [14] and Zhang [24].

We say that $u \in W_{0}^{1, p(x)}(\Omega)$ is a weak solution of the problem (2.7) if

$$
M\left(\int_{\Omega} \frac{1}{p(x)}|\nabla u|^{p(x)}\right) \int_{\Omega}|\nabla u|^{p(x)-2} \nabla u \nabla v=\lambda\left[\int_{\Omega} \frac{1}{q(x)}\left(u^{+}\right)^{q(x)}\right]^{r} \int_{\Omega}\left(u^{+}\right)^{q(x)-1} v,
$$

$\forall v \in W_{0}^{1, p(x)}(\Omega)$.

We define $\tilde{M}, \mathcal{B}$ by

$$
\tilde{M}(u)=M\left(\int_{\Omega} \frac{1}{p(x)}|\nabla u|^{p(x)}\right)
$$

and

$$
\mathcal{B}(u)=\left[\int_{\Omega} \frac{1}{q(x)}|u|^{q(x)}\right]^{r}
$$

and the mappings $T, G, L_{p(x)}, N: W_{0}^{1, p(x)}(\Omega) \rightarrow\left(W_{0}^{1, p(x)}(\Omega)\right)^{*}$ by

$$
\begin{gathered}
T(u)=\tilde{M}(u) \int_{\Omega}|\nabla u|^{p(x)-2} \nabla u \nabla v, \forall u, v \in W_{0}^{1, p(x)}(\Omega), \\
G(u) v=\lambda \mathcal{B}(u) \int_{\Omega}|u|^{q(x)-2} u v, \quad \forall u, v \in W_{0}^{1, p(x)}(\Omega),
\end{gathered}
$$




$$
L_{p(x)}(u) v=\int_{\Omega}|\nabla u|^{p(x)-2} \nabla u \nabla v, \forall u, v \in W_{0}^{1, p(x)}(\Omega)
$$

and

$$
N(u) v=\int_{\Omega}|u|^{q(x)-2} u v, \forall u, v \in W_{0}^{1, p(x)}(\Omega)
$$

So $T(u)=\tilde{M}(u) L_{p(x)}(u)$ and $G(u)=\lambda \mathcal{B}(u) N(u)$ for all $u \in W_{0}^{1, p(x)}(\Omega)$.

THEOREM 2.1 (Fan-Zhang [12]).

(i) $L_{p(x)}: W_{0}^{1, p(x)}(\Omega) \rightarrow\left(W_{0}^{1, p(x)}(\Omega)\right)^{*}$ is a continuous, bounded and strictly monotone operator;

(ii) $L_{p(x)}$ is a mapping of type $S_{+}$, i.e. if $u_{n} \rightarrow u$ in $W_{0}^{1, p(x)}(\Omega)$ and $\lim \sup \left(L_{p(x)}\left(u_{n}\right)-\right.$ $\left.L_{p(x)}(u), u_{n}-u\right) \leq 0$, then $u_{n} \rightarrow u$ in $W_{0}^{1, p(x)}(\Omega) ;$

(iii) $L_{p(x)}: W_{0}^{1, p(x)}(\Omega) \rightarrow\left(W_{0}^{1, p(x)}(\Omega)\right)^{*}$ is a homeomorphism.

It is a standard matter to show that the functional $J_{\lambda}$, described in the Introduction, is of class $C^{1}\left(W_{0}^{1, p(x)}(\Omega), \mathbb{R}\right)$ and

$$
J_{\lambda}^{\prime}(u) v=\tilde{M}(u) \int_{\Omega}|\nabla u|^{p(x)-2} \nabla u \nabla v d x-\lambda\left[\int_{\Omega} \frac{1}{q(x)}\left(u^{+}\right)^{q(x)}\right]^{r} \int_{\Omega}\left(u^{+}\right)^{q(x)-1} v d x,
$$

for all $u, v \in W_{0}^{1, p(x)}(\Omega)$.

3. Main results. This section will be devoted to the proof of the main existing results. Three classes of functions $M$ will be considered and the Mountain Pass Theorem and the Ekeland Variational Principle will be used.

THEOREM 3.1.

(i) Suppose that $1<p(x)<N$ with $1<q(x)<p^{*}(x)=\frac{N p(x)}{N-p(x)}$ for all $x \in \bar{\Omega}$. In addition, assume that exist $0<m_{0}$ and $m_{1}$ such that $m_{0} \leq M(t) \leq m_{1}$, with $\frac{m_{1} p^{+}}{m_{0}}<\frac{\left(q^{-}\right)^{r+1}(r+1)}{\left(q^{+}\right)^{r}}$ and $q^{-}(r+1)>p^{+}$. Then problem (1.1) possesses $a$ weak solution for all $\lambda>0$.

(ii) Suppose that $1<p(x)<N$ with $1<q(x)<p^{*}(x)=\frac{N p(x)}{N-p(x)}$ for all $x \in \bar{\Omega}$. In addition, assume that exist $0<m_{0}$ and $m_{1}$ such that $m_{0} \leq M(t) \leq m_{1}$. If $q^{-}(r+$ 1) $<p^{-}$, there is $\lambda^{*}>0$ such that problem (1.1) possesses a positive solution $u_{\lambda}$ for each $\lambda \in\left(0, \lambda^{*}\right)$.

(iii) Suppose that $1<p(x)<N$ with $1<q(x)<p^{*}(x)=\frac{N p(x)}{N-p(x)}$ for all $x \in \bar{\Omega}$. In addition, assume $M(t)=a+b t$, where $a>0, b>0$ and $t \geq 0$. If $\max \left\{p^{+}, \frac{2\left(p^{+}\right)^{2}}{\left(p^{-}\right)^{2}}\right\}<\frac{(r+1)\left(q^{-}\right)^{r+1}}{\left(q^{+}\right)^{r}}$ and $q^{-}(r+1)>2 p^{+}$, then problem (1.1) possesses a weak positive solution for all $\lambda>0$.

(iv) Suppose that $1<p(x)<N$ with $1<q(x)<p^{*}(x)=\frac{N p(x)}{N-p(x)}$ for all $x \in \bar{\Omega}$ and $M(t)$ as in (iii). If $q^{-}(r+1)<p^{-}$, there is $\lambda^{*}>0$ such that problem (1.1) possesses a positive solution $u_{\lambda}$ for each $\lambda \in\left(0, \lambda^{*}\right)$. 
(v) Suppose that $1<p(x)<N$ with $1<q(x)<p^{*}(x)=\frac{N p(x)}{N-p(x)}$ for all $x \in \bar{\Omega}$. Let $M(t)=t^{\alpha-1}$ be such that $\frac{\alpha\left(p^{+}\right)^{\alpha}}{\left(q^{-}\right)^{\alpha-1}}<\frac{\left(q^{-}\right)^{r+1}(r+1)}{\left(q^{+}\right)^{r}}$ and $q^{-}(r+1)>\alpha p^{+} \geq$ $\alpha p^{-}>1$. Then problem (1.1) possesses a weak positive solution for all $\lambda>0$.

(vi) Suppose that $1<p(x)<N$ with $1<q(x)<p^{*}(x)=\frac{N p(x)}{N-p(x)}$ for all $x \in \bar{\Omega}$. Let $M(t)=t^{\alpha-1}$. If $q^{-}(r+1)<\alpha p^{-}$, there is $\lambda^{*}>0$ such that problem (1.1) possesses a positive solution $u_{\lambda}$ for each $\lambda \in\left(0, \lambda^{*}\right)$.

Proof. (i) Let us use the Mountain Pass Theorem. Recalling that

$$
J_{\lambda}(u)=\widehat{M}\left(\int_{\Omega} \frac{1}{p(x)}|\nabla u|^{p(x)} d x\right)-\frac{\lambda}{r+1}\left[\int_{\Omega} \frac{1}{q(x)}\left(u^{+}\right)^{q(x)} d x\right]^{r+1}
$$

for all $\lambda>0$, we have

$$
J_{\lambda}(u) \geq \frac{m_{0}}{p^{+}} \int_{\Omega}|\nabla u|^{p(x)} d x-\frac{\lambda}{(r+1)\left(q^{-}\right)^{r+1}}\left[\int_{\Omega}|u|^{q(x)}\right]^{r+1} .
$$

We now observe that taking $\|u\|<1$, we obtain $\int_{\Omega}|\nabla u|^{p(x)} d x \geq\|u\|^{p^{+}}$, which implies

$$
J_{\lambda}(u) \geq \frac{m_{0}}{p^{+}}\|u\|^{p^{+}}-\frac{\lambda}{(r+1)\left(q^{-}\right)^{r+1}}\left[\int_{\Omega}|u|^{q(x)}\right]^{r+1} .
$$

Using the Sobolev embedding $W_{0}^{1, p(x)}(\Omega) \hookrightarrow L^{q(x)}(\Omega)$, one has

$$
|u|_{q(x)} \leq C\|u\|=C \rho<1
$$

if $\rho=\|u\|$ is small enough. By Proposition 2.2,

$$
\int_{\Omega}|u|^{q(x)} d x \leq|u|_{q(x)}^{q^{-}}
$$

and so

$$
\left(\int_{\Omega}|u|^{q(x)} d x\right)^{r+1} \leq|u|_{q(x)}^{q^{-(r+1)}}
$$

Thus,

$$
J_{\lambda}(u) \geq \frac{m_{0}}{p^{+}}\|u\|^{p^{+}}-\frac{\lambda}{(r+1)\left(q^{-}\right)^{r+1}}(C\|u\|)^{q^{-}(r+1)}
$$

and taking $\|u\|=\rho$

$$
J_{\lambda}(u) \geq \frac{m_{0}}{p^{+}} \rho^{p^{+}}-\frac{\lambda}{(r+1)\left(q^{-}\right)^{r+1}} C \rho^{q^{-}(r+1)},
$$

which implies

$$
J_{\lambda}(u) \geq \rho^{p^{+}}\left[\frac{m_{0}}{p^{+}}-\frac{\lambda C}{(r+1)\left(q^{-}\right)^{r+1}} \rho^{q^{-}(r+1)-p^{+}}\right] .
$$




\section{FRANCISCO JULIO S. A. CORRÊA AND AUGUSTO CÉSAR DOS REIS COSTA}

Since $q^{-}(r+1)>p^{+}$, we find positive numbers $a, \rho$ such that

$$
J_{\lambda}(u) \geq a>0 \text { if }\|u\|=\rho \text { and for all } \lambda>0 .
$$

We now take $0<\omega \in W_{0}^{1, p(x)}(\Omega)$ and estimate

$$
J_{\lambda}(t \omega)=\widehat{M}\left(\int_{\Omega} \frac{1}{p(x)}|\nabla t \omega|^{p(x)} d x\right)-\frac{\lambda}{r+1}\left[\int_{\Omega} \frac{1}{q(x)}|t \omega|^{q(x)} d x\right]^{r+1} .
$$

For $t>1$, one has $t^{p(x)} \leq t^{p^{+}}$and $t^{q^{-}} \leq t^{q(x)}$ and so

$$
J_{\lambda}(t \omega) \leq \frac{m_{1} t^{p^{+}}}{p^{-}} \int_{\Omega}|\nabla \omega|^{p(x)} d x-\frac{\lambda}{(r+1)} \frac{t^{q^{-}(r+1)}}{\left(q^{+}\right)^{r+1}}\left[\int_{\Omega} \omega^{q(x)} d x\right]^{r+1} .
$$

Using the fact that $q^{-}(r+1)>p^{+}$, we obtain $J_{\lambda}(t \omega) \rightarrow-\infty$ as $t \rightarrow+\infty$. Consequently, $J_{\lambda}$ satisfies the geometry of the Mountain Pass Theorem.

Now to complete the result $(i)$, we show that $J_{\lambda}$ satisfies the Palais-Smale condition (or (PS) for short). This means that all sequence $\left(u_{n}\right) \subset W_{0}^{1, p(x)}(\Omega)$ such that

$$
J_{\lambda}\left(u_{n}\right) \rightarrow C_{\lambda} \text { and } J_{\lambda}^{\prime}\left(u_{n}\right) \rightarrow 0
$$

contains a convergent subsequence in the norm of $W_{0}^{1, p(x)}(\Omega)$.

Let $\left(u_{n}\right) \subset W_{0}^{1, p(x)}(\Omega)$ be a sequence satisfying condition (3.8). Thus, taking

$$
\begin{aligned}
\frac{m_{1} p^{+}}{m_{0}}<\theta<\frac{\left(q^{-}\right)^{r+1}(r+1)}{\left(q^{+}\right)^{r}} & \\
C+\left\|u_{n}\right\| & \geq J_{\lambda}\left(u_{n}\right)-\frac{1}{\theta} J_{\lambda}^{\prime}\left(u_{n}\right) u_{n} \\
& \geq\left(\frac{m_{0}}{p^{+}}-\frac{m_{1}}{\theta}\right) \int_{\Omega}\left|\nabla u_{n}\right|^{p(x)} \\
& +\lambda\left(\frac{1}{\theta\left(q^{+}\right)^{r}}-\frac{1}{\left(q^{-}\right)^{r+1}(r+1)}\right)\left[\int_{\Omega}\left|u_{n}\right|^{q(x)}\right]^{r+1} .
\end{aligned}
$$

If $\left(u_{n}\right)$ is unbounded in $W_{0}^{1, p(x)}(\Omega)$, we may suppose, passing to a subsequence if necessary, that $\left\|u_{n}\right\|>1$ and, in view of the previous inequalities,

$$
C+\left\|u_{n}\right\| \geq\left(\frac{m_{0}}{p^{+}}-\frac{m_{1}}{\theta}\right)\left\|u_{n}\right\|^{p^{-}}
$$

which is an absurd because $p^{-}>1$. Hence, $\left(u_{n}\right)$ is bounded in $W_{0}^{1, p(x)}(\Omega)$. Thus, there exists a subsequence, still denoted by $\left(u_{n}\right)$, such that $u_{n} \rightarrow u$ in $W_{0}^{1, p(x)}(\Omega)$.

From

$$
J_{\lambda}^{\prime}\left(u_{n}\right) \rightarrow 0
$$

we have

$$
J_{\lambda}^{\prime}\left(u_{n}\right)\left(u_{n}-u\right)=\tilde{M}\left(u_{n}\right) \int_{\Omega}\left|\nabla u_{n}\right|^{p(x)-2} \nabla u_{n} \nabla\left(u_{n}-u\right)-G\left(u_{n}\right)\left(u_{n}-u\right) \rightarrow 0 .
$$


By Hölder inequality we obtain

$$
\left.\left.\left|\int_{\Omega}\right| u_{n}\right|^{q(x)-2} u_{n}\left(u_{n}-u\right) d x\left|\leq \int_{\Omega}\right| u_{n}\right|^{q(x)-1}\left|\left(u_{n}-u\right)\right| d x \leq C|u|_{q(x) / q(x)-1}\left|u_{n}-u\right|_{q(x)} .
$$

Since $q(x)<p^{*}(x)$ for all $x \in \bar{\Omega}$, we deduce that $W_{0}^{1, p(x)}(\Omega)$ is compactly embedded in $L^{q(x)}(\Omega)$, hence $\left(u_{n}\right)$ converges strongly to $u$ in $L^{q(x)}(\Omega)$. Then $N\left(u_{n}\right)\left(u_{n}-u\right) \rightarrow 0$.

On the other hand, there exist positive constants $c_{1}$ and $c_{2}$ such that

$$
c_{1} \leq \int_{\Omega} \frac{1}{q(x)}\left|\nabla u_{n}\right|^{q(x)} \leq c_{2} .
$$

So we have $G\left(u_{n}\right)\left(u_{n}-u\right) \rightarrow 0$.

From (3.9) we obtain

$$
L_{p(x)}\left(u_{n}\right)\left(u_{n}-u\right)=\int_{\Omega}\left|\nabla u_{n}\right|^{p(x)-2} \nabla u_{n} \nabla\left(u_{n}-u\right) \rightarrow 0,
$$

since there exists positive constants $c_{3}$ and $c_{3}$ such that $c_{3} \leq \tilde{M}\left(u_{n}\right) \leq c_{4}$. We also have

$$
L_{p(x)}(u)\left(u_{n}-u\right)=\int_{\Omega}|\nabla u|^{p(x)-2} \nabla u \nabla\left(u_{n}-u\right) \rightarrow 0 .
$$

Consequently,

$$
\left(L_{p(x)}\left(u_{n}\right)-L_{p(x)}(u), u_{n}-u\right) \rightarrow 0 .
$$

From Theorem 2.1, we have $u_{n} \rightarrow u$ in $W_{0}^{1, p(x)}(\Omega)$ and the proof of $(i)$ is over.

(ii) We will use the Ekeland Variational Principle following the ideas contained in Mihailescu and Radulescu [19]. Reasoning as before, we obtain

$$
J_{\lambda}(u) \geq \frac{m_{0}}{p^{+}} \int_{\Omega}|\nabla u|^{p(x)} d x-\frac{\lambda}{(r+1)\left(q^{-}\right)^{r+1}}\left[\int_{\Omega}|u|^{q(x)}\right]^{r+1},
$$

and so for $\|u\|=\rho$ sufficiently small

$$
J_{\lambda}(u) \geq \rho^{q^{-}(r+1)}\left[\frac{m_{0} \rho^{p^{+}-q^{-}(r+1)}}{p^{+}}-\frac{\lambda C}{(r+1)\left(q^{-}\right)^{r+1}}\right] \geq a>0 \text { if } 0<\lambda<\lambda^{*}
$$

for some $\lambda^{*}>0$. Thus, for all $\lambda \in\left(0, \lambda^{*}\right)$, we have

$$
\inf _{\partial B_{\rho}(0)} J_{\lambda}>0
$$

Note that in this item the parameter $\lambda$ plays a crucial role.

Now let $\epsilon_{0}>0$ be such that $q^{-}(r+1)+\epsilon_{0}(r+1)<p^{-}$. Because $q \in C(\bar{\Omega})$, it follows that there exists an open set $\Omega_{0} \subset \Omega$ such that $\left|q(x)-q^{-}\right|<\epsilon_{0}$ for all $x \in \Omega_{0}$. Thus, we conclude that $q(x)(r+1) \leq q^{-}(r+1)+\epsilon_{0}(r+1)<p^{-}$for all $x \in \Omega_{0}$.

Let $\phi \in C_{0}^{\infty}(\Omega)$ be such that $\operatorname{supp} \phi \supset \bar{\Omega}_{0}, \phi(x)=1$ for all $x \in \Omega_{0}$ and $0 \leq \phi \leq 1$ in $\Omega$. For $t$ sufficiently small, $t \in(0,1)$, we have that $t \phi \in B_{\rho}(0)$, where $B_{\rho}(0)$ is the ball 
centred at the origin and of radius $\rho$ in $W_{0}^{1, p(x)}(\Omega)$, and

$$
\begin{aligned}
J_{\lambda}(t \phi) & \leq \frac{m_{1} t^{p^{-}}}{p^{-}} \int_{\Omega}|\nabla \phi|^{p(x)} d x-\frac{\lambda}{(r+1)} \frac{1}{\left(q^{+}\right)^{r+1}}\left[\int_{\Omega}|t \phi|^{q(x)} d x\right]^{r+1} \\
& \leq \frac{m_{1} t^{p^{-}}}{p^{-}} \int_{\Omega}|\nabla \phi|^{p(x)} d x-\frac{\lambda}{(r+1)} \frac{t^{q^{-}(r+1)+\epsilon_{0}(r+1)}}{\left(q^{+}\right)^{r+1}}\left[\int_{\Omega_{0}}|\phi|^{q(x)} d x\right]^{r+1} .
\end{aligned}
$$

Therefore, in view of $\int_{\Omega}|\nabla \phi|^{p(x)} d x>0$, we get

$$
J_{\lambda}(t \phi)<0
$$

Since for all $u \in B_{\rho}(0)$ we get

$$
J_{\lambda}(u) \geq \frac{m_{0}}{p^{+}}\|u\|^{p^{+}}-\frac{\lambda C}{r+1} \frac{1}{\left(q^{-}\right)^{r+1}}\|u\|^{q^{-(r+1)}},
$$

it follows that

$$
-\infty<\underline{c}:=\inf _{\overline{B_{\rho}}(0)} J_{\lambda}<0
$$

We now let $0<\epsilon<\inf _{\partial B_{\rho}(0)} J_{\lambda}-\inf _{B_{\rho}(0)} J_{\lambda}$. Applying Ekeland's Variational Principle to the functional $J_{\lambda}: \bar{B}_{\rho}(0) \rightarrow \mathbb{R}$, we find $u_{\epsilon} \in \bar{B}_{\rho}(0)$ such that

$$
J_{\lambda}\left(u_{\epsilon}\right)<\inf _{B_{\rho}(0)} J_{\lambda}+\epsilon \text { and } J_{\lambda}\left(u_{\epsilon}\right)<J_{\lambda}(u)+\epsilon\left\|u-u_{\epsilon}\right\|, u \neq u_{\epsilon} .
$$

Reasoning as in Mihailescu and Radulescu [19], we obtain $\left(w_{n}\right) \subset B_{\rho}(0)$ such that

$$
J_{\lambda}\left(w_{n}\right) \rightarrow \underline{c} \text { and } J_{\lambda}^{\prime}\left(w_{n}\right) \rightarrow 0 .
$$

Since $q(x)<p^{*}(x)$ and $J_{\lambda}$ satisfy the (PS) condition, we have a subsequence, denoted still by $w_{n}$, and an element $w \in W_{0}^{1, p}(\Omega)$ such that $w_{n} \rightarrow w, J_{\lambda}(w)=\underline{c}<0$ and $J_{\lambda}^{\prime}(w)=0$, and thus we conclude that $w$ is a nontrivial weak solution for problem (1.1).

(iii) Note that

$$
J_{\lambda}(u)=a\left(\int_{\Omega} \frac{1}{p(x)}|\nabla u|^{p(x)} d x\right)+\frac{b}{2}\left(\int_{\Omega} \frac{1}{p(x)}|\nabla u|^{p(x)} d x\right)^{2}-\frac{\lambda}{r+1}\left[\int_{\Omega} \frac{1}{q(x)}\left(u^{+}\right)^{q(x)} d x\right]^{r+1}
$$

for all $\lambda>0$ and

$$
J_{\lambda}(u) \geq \frac{a}{p^{+}} \int_{\Omega}|\nabla u|^{p(x)} d x+\frac{b}{2\left(p^{+}\right)^{2}}\left(\int_{\Omega}|\nabla u|^{p(x)} d x\right)^{2}-\frac{\lambda}{(r+1)\left(q^{-}\right)^{r+1}}\left[\int_{\Omega}|u|^{q(x)}\right]^{r+1} .
$$

We now observe that taking $\|u\|<1$,

$$
J_{\lambda}(u) \geq \frac{a}{p^{+}}\|u\|^{p^{+}}+\frac{b}{2\left(p^{+}\right)^{2}}\|u\|^{2 p^{+}}-\frac{\lambda}{(r+1)\left(q^{-}\right)^{r+1}}\left[\int_{\Omega}|u|^{q(x)}\right]^{r+1} .
$$


Thus,

$$
J_{\lambda}(u) \geq \frac{a}{p^{+}}\|u\|^{p^{+}}+\frac{b}{2\left(p^{+}\right)^{2}}\|u\|^{2 p^{+}}-\frac{\lambda}{(r+1)\left(q^{-}\right)^{r+1}}(C\|u\|)^{q^{-}(r+1)} .
$$

Since $q^{-}(r+1)>2 p^{+}$, we find positive numbers $\delta, \rho$ such that

$$
J_{\lambda}(u) \geq \delta>0 \text { if }\|u\|=\rho \text { and for all } \lambda>0 .
$$

Using arguments as in (i), we find $0<\omega \in W_{0}^{1, p(x)}(\Omega)$ such that $J_{\lambda}(t \omega) \rightarrow-\infty$ as $t \rightarrow+\infty$. Consequently, $J_{\lambda}$ satisfies the geometry of the Mountain Pass Theorem.

It rests to show that $J_{\lambda}$ enjoys the (PS) condition.

Let $\left(u_{n}\right) \subset W_{0}^{1, p(x)}(\Omega)$ be a sequence such that

$$
J_{\lambda}\left(u_{n}\right) \rightarrow C_{\lambda} \text { and } J_{\lambda}^{\prime}\left(u_{n}\right) \rightarrow 0 .
$$

Thus, taking

$$
\max \left\{p^{+}, \frac{2\left(p^{+}\right)^{2}}{\left(p^{-}\right)^{2}}\right\}<\theta<\frac{(r+1)\left(q^{-}\right)^{r+1}}{\left(q^{+}\right)^{r}}
$$

we have

$$
\begin{aligned}
C+\left\|u_{n}\right\| \geq & J_{\lambda}\left(u_{n}\right)-\frac{1}{\theta} J_{\lambda}^{\prime} u_{n} u_{n} \\
\geq & a\left(\int_{\Omega} \frac{1}{p(x)}\left|\nabla u_{n}\right|^{p(x)}\right)+\frac{b}{2}\left(\int_{\Omega} \frac{1}{p(x)}\left|\nabla u_{n}\right|^{p(x)}\right)^{2} \\
& -\frac{\lambda}{r+1}\left[\int_{\Omega} \frac{1}{q(x)}|u|^{q(x)}\right]^{r+1}-\frac{a}{\theta}\left(\int_{\Omega}\left|\nabla u_{n}\right|^{p(x)}\right) \\
& -\frac{b}{\theta}\left(\int_{\Omega} \frac{1}{p(x)}\left|\nabla u_{n}\right|^{p(x)}\right)^{2}+\frac{\lambda}{\theta\left(q^{+}\right)^{r}}\left[\int_{\Omega}|u|^{q(x)}\right]^{r+1} \\
\geq & \left(\frac{a}{p^{+}}-\frac{a}{\theta}\right)\left(\int_{\Omega}\left|\nabla u_{n}\right|^{p(x)}\right)+\left(\frac{b}{2\left(p^{+}\right)^{2}}-\frac{b}{\theta\left(p^{-}\right)^{2}}\right)\left(\int_{\Omega}\left|\nabla u_{n}\right|^{p(x)}\right)^{2} \\
& +\lambda\left(\frac{1}{\theta\left(q^{+}\right)^{r}}-\frac{1}{(r+1)\left(q^{-}\right)^{r+1}}\right)\left[\int_{\Omega}|u|^{q(x)}\right]^{r+1} .
\end{aligned}
$$

Now suppose that $\left(u_{n}\right)$ is unbounded in $W_{0}^{1, p(x)}(\Omega)$. Thus, passing to a subsequence if necessary, we get $\left\|u_{n}\right\|>1$ and

$$
C+\left\|u_{n}\right\| \geq\left(\frac{a}{p^{+}}-\frac{a}{\theta}\right)\left\|u_{n}\right\|^{p^{+}}+\left(\frac{b}{2\left(p^{+}\right)^{2}}-\frac{b}{\theta\left(p^{-}\right)^{2}}\right)\left\|u_{n}\right\|^{2 p^{+}},
$$

which is an absurd because $p^{+}>1$. Hence, $\left(u_{n}\right)$ is bounded in $W_{0}^{1, p(x)}(\Omega)$. Thus, there exists a subsequence, still denoted by $u_{n}$, such that $u_{n} \rightarrow u$ in $W_{0}^{1, p(x)}(\Omega)$.

From

$$
J_{\lambda}^{\prime}\left(u_{n}\right) \rightarrow 0
$$


we have

$$
J_{\lambda}^{\prime}\left(u_{n}\right)\left(u_{n}-u\right)=\left(a+b \int_{\Omega} \frac{1}{p(x)}\left|\nabla u_{n}\right|^{p(x) d x}\right) L_{p(x)} u_{n}\left(u_{n}-u\right)-G\left(u_{n}\right)\left(u_{n}-u\right) \rightarrow 0 .
$$

Reasoning as in the proof of $(i)$, we obtain $G\left(u_{n}\right)\left(u_{n}-u\right) \rightarrow 0$, positive constants $c_{3}$ and $c_{4}$ such that $c_{3} \leq \int_{\Omega} \frac{1}{p(x)}\left|\nabla u_{n}\right|^{p(x)} \leq c_{4}$ and

$$
L_{p(x)}\left(u_{n}\right)\left(u_{n}-u\right)=\int_{\Omega}\left|\nabla u_{n}\right|^{p(x)-2} \nabla u_{n} \nabla\left(u_{n}-u\right) \rightarrow 0 .
$$

We also have

$$
L_{p(x)}(u)\left(u_{n}-u\right)=\int_{\Omega}|\nabla u|^{p(x)-2} \nabla u \nabla\left(u_{n}-u\right) \rightarrow 0 .
$$

Consequently,

$$
\left(L_{p(x)}\left(u_{n}\right)-L_{p(x)}(u), u_{n}-u\right) \rightarrow 0 .
$$

From Theorem 2.1 we have $u_{n} \rightarrow u$ in $W_{0}^{1, p(x)}(\Omega)$, and the proof of (iii) is over.

(iv) Reasoning as before, we obtain with $\|u\|<1$

$$
J_{\lambda}(u) \geq \frac{a}{p^{+}}\|u\|^{p^{+}}+\frac{b}{2\left(p^{+}\right)^{2}}\|u\|^{2 p^{+}}-\frac{\lambda}{(r+1)\left(q^{-}\right)^{r+1}}(C\|u\|)^{q^{-}(r+1)}
$$

and so for $\|u\|=\rho$ sufficiently small

$$
J_{\lambda}(u) \geq \delta>0 \text { if } 0<\lambda<\lambda^{*} .
$$

We can take $\lambda^{*}=\frac{1}{3} \min \left\{\frac{(r+1)\left(q^{-}\right)^{r+1} a(\rho)^{p^{+}-q^{-}(r+1)}}{C p^{+}}, \frac{(r+1)\left(q^{-}\right)^{r+1} b(\rho)^{2 p^{+}-q^{-}(r+1)}}{2 C\left(p^{+}\right)^{2}}\right\}$.

Thus, for all $\lambda \in\left(0, \lambda^{*}\right)$, we have

$$
\inf _{\partial B_{\rho}(0)} J_{\lambda}>0
$$

Following (ii) for $t$ sufficiently small, $t \in(0,1)$, we have that $t \phi \in B_{\rho}(0)$ such that

$$
\begin{aligned}
& J_{\lambda}(t \phi) \leq \frac{a t^{p^{-}}}{p^{-}} \int_{\Omega}|\nabla \phi|^{p(x)} d x+\frac{b t^{2 p^{-}}}{2\left(p^{-}\right)^{2}} \int_{\Omega}|\nabla \phi|^{p(x)} d x-\frac{\lambda}{(r+1)} \frac{1}{\left(q^{+}\right)^{r+1}}\left[\int_{\Omega}|t \phi|^{q(x)} d x\right]^{r+1} \\
& \leq \frac{a t^{p^{-}}}{p^{-}} \int_{\Omega}|\nabla \phi|^{p(x)} d x+\frac{b t^{2 p^{-}}}{2\left(p^{-}\right)^{2}} \int_{\Omega}|\nabla \phi|^{p(x)} d x-\frac{\lambda}{(r+1)} \frac{t^{q^{-}(r+1)+\epsilon_{0}(r+1)}}{\left(q^{+}\right)^{r+1}}\left[\int_{\Omega_{0}}|\phi|^{q(x)} d x\right]^{r+1} .
\end{aligned}
$$

Therefore, in view of $\int_{\Omega}|\nabla \phi|^{p(x)} d x>0$, we get

$$
J_{\lambda}(t \phi)<0
$$


Since, for all $u \in B_{\rho}(0)$ we get,

$$
J_{\lambda}(u) \geq \frac{a}{p^{+}}\|u\|^{p^{+}}+\frac{b}{2\left(p^{+}\right)^{2}}\|u\|^{2 p^{+}}-\frac{\lambda C}{r+1} \frac{1}{\left(q^{-}\right)^{r+1}}\|u\|^{q^{-(r+1)}},
$$

it follows that

$$
-\infty<\underline{c}:=\inf _{B_{\rho}(0)} J_{\lambda}<0 .
$$

Applying Ekeland's Variational Principle to the functional $J_{\lambda}: \bar{B}_{\rho}(0) \rightarrow \mathbb{R}$, and reasoning as Mihailescu and Radulescu [19], we have $\left(w_{n}\right) \subset B_{\rho}(0)$ such that

$$
J_{\lambda}\left(w_{n}\right) \rightarrow \underline{c} \text { and } J_{\lambda}^{\prime}\left(w_{n}\right) \rightarrow 0 .
$$

Thus, we conclude that $w$ is a nontrivial weak solution for problem (1.1).

(v) We will use the Mountain Pass Theorem. Recalling that

$$
J_{\lambda}(u)=\frac{1}{\alpha}\left(\int_{\Omega} \frac{1}{p(x)}|\nabla u|^{p(x)} d x\right)^{\alpha}-\frac{\lambda}{r+1}\left[\int_{\Omega} \frac{1}{q(x)}\left(u^{+}\right)^{q(x)} d x\right]^{r+1}
$$

for all $\lambda>0$, we have

$$
J_{\lambda}(u) \geq \frac{1}{\alpha\left(p^{+}\right)^{\alpha}}\left(\int_{\Omega}|\nabla u|^{p(x)} d x\right)^{\alpha}-\frac{\lambda}{(r+1)\left(q^{-}\right)^{r+1}}\left[\int_{\Omega}|u|^{q(x)}\right]^{r+1} .
$$

Following $(i)$ and taking $\|u\|=\rho$, we have

$$
J_{\lambda}(u) \geq \frac{1}{\alpha p^{+}} \rho^{\alpha p^{+}}-\frac{\lambda}{(r+1)\left(q^{-}\right)^{r+1}} C \rho^{q^{-}(r+1)},
$$

which implies

$$
J_{\lambda}(u) \geq \rho^{\alpha p^{+}}\left[\frac{1}{\alpha p^{+}}-\frac{\lambda C}{(r+1)\left(q^{-}\right)^{r+1}} \rho^{q^{-}(r+1)-\alpha p^{+}}\right] .
$$

Since $q^{-}(r+1)>\alpha p^{+}$, we find positive numbers $a, \rho$ such that

$$
J_{\lambda}(u) \geq a>0 \text { if }\|u\|=\rho \text { and for all } \lambda>0 .
$$

By the same arguments, corresponding to (i) we take $0<\omega \in W_{0}^{1, p(x)}(\Omega)$ and using the fact that $q^{-}(r+1)>\alpha p^{+}$, we obtain $J_{\lambda}(t \omega) \rightarrow-\infty$ as $t \rightarrow+\infty$. Consequently, $J_{\lambda}$ satisfies the geometry of the Mountain Pass Theorem.

Note that $J_{\lambda}$ enjoys the (PS) condition. Let $\left(u_{n}\right) \subset W_{0}^{1, p(x)}(\Omega)$ be a sequence such that

$$
J_{\lambda}\left(u_{n}\right) \rightarrow C_{\lambda} \text { and } J_{\lambda}^{\prime}\left(u_{n}\right) \rightarrow 0 .
$$

Thus, taking $\frac{\alpha\left(p^{+}\right)^{\alpha}}{\left(q^{-}\right)^{\alpha-1}}<\theta<\frac{\left(q^{-}\right)^{r+1}(r+1)}{\left(q^{+}\right)^{r}}$ 


$$
\begin{aligned}
C+\left\|u_{n}\right\| & \geq J_{\lambda}\left(u_{n}\right)-\frac{1}{\theta} J_{\lambda}^{\prime}\left(u_{n}\right) u_{n} \\
& \geq\left(\frac{1}{\alpha\left(p^{-}\right)^{\alpha}}-\frac{1}{\theta\left(q^{-}\right)^{\alpha-1}}\right)\left(\int_{\Omega}\left|\nabla u_{n}\right|^{p(x)}\right)^{\alpha} \\
& +\lambda\left(\frac{1}{\theta\left(q^{+}\right)^{r}}-\frac{1}{\left(q^{-}\right)^{r+1}(r+1)}\right)\left[\int_{\Omega}\left|u_{n}\right|^{q(x)}\right]^{r+1} .
\end{aligned}
$$

Now suppose that $\left(u_{n}\right)$ is unbounded in $W_{0}^{1, p(x)}(\Omega)$. Thus, passing to a subsequence if necessary, we get $\left\|u_{n}\right\|>1$ and

$$
C+\left\|u_{n}\right\| \geq\left(\frac{1}{\alpha\left(p^{-}\right)^{\alpha}}-\frac{1}{\theta\left(q^{-}\right)^{\alpha-1}}\right)\left\|u_{n}\right\|^{\alpha p^{-}}
$$

which is an absurd because $\alpha p^{-}>1$. Hence, $\left(u_{n}\right)$ is bounded in $W_{0}^{1, p(x)}(\Omega)$. Thus, there exists a subsequence, still denoted by $u_{n}$, such that $u_{n} \rightarrow u$ in $W_{0}^{1, p(x)}(\Omega)$.

From

$$
J_{\lambda}^{\prime}\left(u_{n}\right) \rightarrow 0
$$

we have

$$
J_{\lambda}^{\prime}\left(u_{n}\right)\left(u_{n}-u\right)=\left(\int_{\Omega} \frac{1}{p(x)}\left|\nabla u_{n}\right|^{p(x) d x}\right)^{\alpha-1} L_{p(x)} u_{n}\left(u_{n}-u\right)-G\left(u_{n}\right)\left(u_{n}-u\right) \rightarrow 0 .
$$

Doing an argument similar to that used in the proof of $(i)$, we obtain $G\left(u_{n}\right)\left(u_{n}-u\right) \rightarrow 0$, positive constants $c_{3}$ e $c_{4}$ such that $c_{3} \leq \int_{\Omega} \frac{1}{p(x)}\left|\nabla u_{n}\right|^{p(x)} \leq c_{4}$ and

$$
L_{p(x)}\left(u_{n}\right)\left(u_{n}-u\right)=\int_{\Omega}\left|\nabla u_{n}\right|^{p(x)-2} \nabla u_{n} \nabla\left(u_{n}-u\right) \rightarrow 0 .
$$

We also have

$$
L_{p(x)}(u)\left(u_{n}-u\right)=\int_{\Omega}|\nabla u|^{p(x)-2} \nabla u \nabla\left(u_{n}-u\right) \rightarrow 0 .
$$

Consequently,

$$
\left(L_{p(x)}\left(u_{n}\right)-L_{p(x)}(u), u_{n}-u\right) \rightarrow 0 .
$$

From Theorem 2.1 we have $u_{n} \rightarrow u$ em $W_{0}^{1, p(x)}(\Omega)$ and the proof of $(v)$ is over.

(vi) Following (ii) we obtain, for $\|u\|<1$,

$$
J_{\lambda}(u) \geq \frac{1}{\alpha\left(p^{+}\right)^{\alpha}}\|u\|^{\alpha p^{+}}-\frac{\lambda}{(r+1)\left(q^{-}\right)^{r+1}}(C\|u\|)^{q^{-}(r+1)}
$$


and so for $\|u\|=\rho$ sufficiently small

$$
J_{\lambda}(u) \geq \delta>0 \text { if } 0<\lambda<\lambda^{*}
$$

for some $\lambda^{*}>0$. Thus, for all $\lambda \in\left(0, \lambda^{*}\right)$ we have

$$
\inf _{\partial B_{\rho}(0)} J_{\lambda}>0
$$

By the same arguments, corresponding to (ii) for $t$ sufficiently small, $t \in(0,1)$, we have $t \phi \in B_{\rho}(0)$ such that

$$
\begin{aligned}
& J_{\lambda}(t \phi) \leq \frac{t^{\alpha p^{-}}}{\alpha\left(p^{-}\right)^{\alpha}} \int_{\Omega}|\nabla \phi|^{p(x)} d x-\frac{\lambda}{(r+1)} \frac{1}{\left(q^{+}\right)^{r+1}}\left[\int_{\Omega}|t \phi|^{q(x)} d x\right]^{r+1} \\
& \leq \frac{t^{\alpha p^{-}}}{\alpha\left(p^{-}\right)^{\alpha}} \int_{\Omega}|\nabla \phi|^{p(x)} d x-\frac{\lambda}{(r+1)} \frac{t^{q^{-}(r+1)+\epsilon_{0}(r+1)}}{\left(q^{+}\right)^{r+1}}\left[\int_{\Omega_{0}}|\phi|^{q(x)} d x\right]^{r+1} .
\end{aligned}
$$

Therefore, in view of $\int_{\Omega}|\nabla \phi|^{p(x)} d x>0$, we get

$$
J_{\lambda}(t \phi)<0
$$

Since, for all $u \in B_{\rho}(0)$ we get

$$
J_{\lambda}(u) \geq \frac{1}{\alpha\left(p^{+}\right)^{\alpha}}\|u\|^{\alpha p^{+}}-\frac{\lambda}{(r+1)\left(q^{-}\right)^{r+1}}(C\|u\|)^{q^{-}(r+1)},
$$

it follows that

$$
-\infty<\underline{c}:=\inf _{B_{\rho}(0)} J_{\lambda}<0
$$

Applying Ekeland's Variational Principle to the functional $J_{\lambda}: \bar{B}_{\rho}(0) \rightarrow \mathbb{R}$, and reasoning as Mihailescu and Radulescu [19], we have $\left(w_{n}\right) \subset B_{\rho}(0)$ such that

$$
J_{\lambda}\left(w_{n}\right) \rightarrow \underline{c} \text { and } J_{\lambda}^{\prime}\left(w_{n}\right) \rightarrow 0
$$

Consequently $w$ is a nontrivial weak solution for problem (1.1).

REMARK. We present below Table 1 summarizing our results. 
Table 1. Results

\begin{tabular}{|c|c|c|c|c|}
\hline$M(t)$ & & Hypotheses & $\mathrm{MPT}^{a}$ & $\mathrm{EVP}^{b}$ \\
\hline$m_{0} \leq M(t) \leq m_{1}$ & & $\begin{array}{l}\frac{m_{1} p^{+}}{m_{0}}<\frac{\left(q^{-}\right)^{r+1}(r+1)}{\left(q^{+}\right)^{r}} \\
\text { and } q^{-}(r+1)>p^{+}\end{array}$ & $\begin{array}{c}\text { Yes } \\
\text { for all } \lambda>0\end{array}$ & Not used \\
\hline$m_{0} \leq M(t) \leq m_{1}$ & & $q^{-}(r+1)<p^{-}$ & No & Yes for $\lambda \in\left(0, \lambda^{*}\right)$ \\
\hline$M(t)=a+b t$ & $\max$ & $\left\{\begin{aligned}\left.p^{+}, \frac{2\left(p^{+}\right)^{2}}{\left(p^{-}\right)^{2}}\right\}<\frac{(r+1)\left(q^{-}\right)^{r+1}}{\left(q^{+}\right)^{r}} & <\text { and } q^{-}(r+1)>2 p^{+}\end{aligned}\right.$ & $\begin{array}{c}\text { Yes } \\
\text { for all } \lambda>0\end{array}$ & Not used \\
\hline$M(t)=a+b t$ & & $q^{-}(r+1)<p^{-}$ & No & Yes for $\lambda \in\left(0, \lambda^{*}\right)$ \\
\hline$M(t)=t^{\alpha-1}$ & & $\begin{array}{l}\frac{\alpha\left(p^{+}\right)^{\alpha}}{\left(q^{-}\right)^{\alpha-1}}<\frac{\left(q^{-}\right)^{r+1}(r+1)}{\left(q^{+}\right)^{r}} \\
\quad \text { and } q^{-}(r+1)>p^{+}\end{array}$ & $\begin{array}{c}\text { Yes } \\
\text { for all } \lambda>0\end{array}$ & Not used \\
\hline$M(t)=t^{\alpha-1}$ & & $q^{-}(r+1)<\alpha p^{-}$ & No & Yes for $\lambda \in\left(0, \lambda^{*}\right)$ \\
\hline
\end{tabular}

${ }^{a}$ MPT: Existence of solution via The Mountain Pass Theorem.

${ }^{b}$ EVP: Existence of solution via Ekeland's Variational Principle.

ACKNOWLEDGEMENTS. This work was done while the first author was visiting the Postgraduate Program in Mathematics of the Universidade Federal do Pará, Brazil. He would like to express his warm gratitude to its staff, particularly to Professors Giovany M. Figueiredo and Rúbia G. Nascimento, for the hospitality and friendship. The first author is partially supported by CNPq-Brazil under Grant 300561/2010-5. The authors would like to thank an anonymous referee whose comments and suggestions contributed towards the improvement of this work.

\section{REFERENCES}

1. R. P. Agarwal, K. Perera and Z. Zhang, On some non-local eigenvalue problems, Discrete Contin. Dyn. Syst. Ser. S 5(4) (August 2012), 707-714; doi:10.3934/dcdss.2012.5.707.

2. C. O Alves, F. J. S. A. Corrêa and T. F. Ma, Positive solutions for a quasilinear elliptic equation of Kirchhoff type, Comput. Math. Appl. 49 (2005), 85-93.

3. J. W. Bebernes and A. Lacey, Global existence and finite-time blow-up for a class of nonlocal parabolic problems, Adv. Differ. Equ. 2(6) (1997), 927-953.

4. J. W. Bebernes and P. Talaga, Nonlocal problems modelling shear banding, Comm. Appl. Nonlinear Anal. 3 (2) (1996), 79-103.

5. T. J. Burns, On a combustion-like model for plastic strain localization, Chapter 2, in Shock induced transitions and phase structure in general media (Fosdick, R. et al., Editors) (Springer-Verlag, New York, 1992). 261-271.

6. T. J. Burns, Does a shear band result from a thermal explosion?, Mech. Mater. 17 (1994),

7. E. Caglioti, P. L. Lions, C. Marchiori and M. Pulvirenti, A special class of stationary flows for two - dimensional Euler equations: A statistical mechanics description, Comm. Math. Phys. 143 (1992), 501-525.

8. J. A. Carrillo, On a nonlocal elliptic equation with decreasing nonlinearity arising in plasma physics and heat conduction, Nonlinear Anal. 32 (1) (1998), 97-115.

9. F. J. S. A. Corrêa and G. M. Figueiredo, On an elliptic equation of p-Kirchhof type via variational methods, Bull. Aust. Math. Soc. 74 (2006), 263-277.

10. J. Dolbeault, Stationary states in plasma physics: Maxwellian solutions of the VlasovPoisson system, Math. Models. Meth. Appl. Sci. 1 (1991), 183-148.

11. X. L. Fan, J. S. Shen and D. Zhao, Sobolev embedding theorems for spaces $W^{k, p(x)}(\Omega)$, J. Math. Anal. Appl. 262 (2001), 749-760. 
12. X. L. Fan and Q. H. Zhang, Existence of solutions for $\mathrm{p}(\mathrm{x})$-Laplacian Dirichlet problems, Nonlinear Anal. 52 (2003), 1843-1852.

13. X. L. Fan and D. Zhao, On the spaces $L^{p(x)}$ and $W^{m, p(x)}$, J. Math. Anal. Appl. 263 (2001), $424-446$.

14. X. Fan, Y. Zhao and Q. Zhang, A strong maximum principle for $\mathrm{p}(\mathrm{x})$-Laplace equations, Chin. J. Contemp. Math. 21 (1) (2000), 1-7.

15. D. Gogny and P. L. Lions, Sur les états déqulibre pour las densités életroniques das les plasmas, RAIRO Modél. Math. Anal. Numér. 23 (1998), 137-153.

16. J. M. Gomes and L. Sanchez, On a variational approach to some non-local boundary value problems, Appl. Anal., 84 (9) (September 2005), 909-925.

17. A. Krzywick and T. Nadzieja, Some results concerning the Poisson-Boltzmann equation, Zastosowania Math. Appl. Math., 21 (2) (1991), 265-272.

18. M. Mihailescu and V. Radulescu, A multiplicity result for a nonlinear degenerate problem arising in the theory of electrorheological fluids, Proc. R. Soc. A, 462 (2006), 2625-2641.

19. M. Mihailescu and V. Radulescu, On a non-homogeneous quasilinear eigenvalue problem in Sobolev spaces with variable exponent, Proc. Amer. Math. Soc., 135 (9) (September 2007), 2929-2937.

20. W. E. Olmstead, S. Nemat-Nasser and L. Ni, Shear bands as surfaces of discontinuity, J. Mech. Phys. Solids, 42 (1994), 697-709.

21. K. Perera and Z. Zhang, Nontrivial solutions of Kirchhoff-type problems via Yang index, J. Diff. Equ. 221 (2006), 246-255.

22. K. Perera and Z. Zhang, Sign changing solutions of Kirchhoff type problems via invariant sets of descent flows, J. Math. Anal. Appl. 317 (2006), 456-463.

23. M. Rúžička, Electrorheological fluids: modelling and mathematical theory (SpringerVerlag, Berlin, Germany, 2000).

24. Q. Zhang, A strong maximum principle for differential equations with non-standard $p(x)$-growth conditions, J. Math. Anal. Appl. 312 (2005), 24-32. 